\title{
Individualisierte Tumortherapie und Status quo beim Melanom
}

\author{
Sebastian Bauer ${ }^{a *}$ Heinz Schmidberger ${ }^{b *} \quad$ Uwe Trefzer $^{c *}$

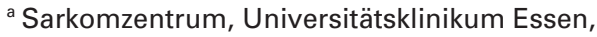 \\ ${ }^{\mathrm{b}}$ Klinik und Poliklinik für Radioonkologie und Strahlentherapie, Universitätsmedizin Mainz, \\ ${ }^{\mathrm{c}}$ Klinik für Dermatologie, Venerologie und Allergologie, Hauttumorcentrum Charité - Universitätsmedizin Berlin, Deutschland
}

\section{Neuentwicklungen beim metastasierten Melanom}

Nach über 30 Jahren des therapeutischen Stillstands konnte die Prognose beim metastasierten Melanom in letzter Zeit durch medikamentöse Neuentwicklungen deutlich verbessert werden. 2011 wurde der vollständig humanisierte, monoklonale Anti-cytotoxic T lymphocyte antigen-4 (CTLA-4)-Antikörper Ipilimumab in den Markt eingeführt, die Zulassung des BRAF-Inhibitors Vemurafenib wird 2012 erwartet.

\section{Immuntherapie}

\section{CTLA-4-Blockade}

Da im B16-Melanom-Modell die antitumorale Wirksamkeit einer CTLA-4-Blockade durch Kombination mit einer Tumorvakzine deutlich verstärkt werden konnte [1], wurde Ipilimumab in der 3-armigen Studie MDX010-20 mit der Glykoprotein (gp)100-Tumorvakzine kombiniert. Anders als im Tiermodell war in der für die Zulassung von Ipilimumab als Monotherapie relevanten Phase-III-Studie die Kombination des CTLA-4-Antikörpers mit gp100 aber nicht wirksamer als die Ipilimumab-Monotherapie, die das Mortalitätsrisiko um $34 \%$ reduzierte (Abb. 1) [2].

Als Gründe für den fehlenden Zusatznutzen der gp100Vakzine werden die Auswahl des Epitops und das in diesem Fall möglicherweise ungeeignete Freund's Adjuvans diskutiert. Für die Kombination von gp100 mit Interleukin-2 wurde zwar eine Antitumorwirkung gezeigt, im Vergleich zu einer Interleukin-Monotherapie konnte das mediane Gesamtüberleben beim fortgeschrittenen Melanom aber nicht signifikant verbessert werden [3].

Die Suche nach einem therapeutisch nutzbaren Surrogatmarker gestaltet sich bei Ipilimumab schwierig. Als Prädiktor für einen Erfolg der Ipilimumab-Therapie wird unter anderem

\footnotetext{
*Alle Autoren haben zu gleichen Teilen zu diesem Artikel beigetragen.
}

das auf aktivierten T-Zellen exprimierte Oberflächenmolekül ICOS (induzierbarer Kostimulator) untersucht. Nachdem zunächst gezeigt werden konnte, dass bei einer CTLA-4-Blockade die Zahl der ICOS exprimierenden T-Zellen steigt, weisen die Ergebnisse neuer tierexperimenteller Studien darauf hin, dass der ICOS/ICOS-Ligand (ICOSL)-Signalweg zugleich eine wichtige Voraussetzung für die optimale antitumorale Wirksamkeit der CTLA-4-Blockade ist. Im Vergleich zu der bei Mäusen mit Wildtyp-Tumoren $\left(\mathrm{ICOS}^{+}\right)$ausgelösten antitumoralen Antwort war die Wirksamkeit der CTLA-4-Blockade bei ICOS$^{-}$- und $\mathrm{ICOSL}^{-}$-Mäusen mit B16/BL6-Melanomen deutlich vermindert [4]. Inwiefern sich ICOS als Verlaufsparameter für die Praxis eignet, muss noch untersucht werden.

Als weiterer möglicher Surrogatmarker für ein längeres Gesamtüberleben bei der Therapie mit Ipilimumab erwies sich in einer US-amerikanischen Studie die Lymphozytenzahl im frühen Behandlungsverlauf. Die Auswertung der Überlebensdaten von 51 Patienten mit fortgeschrittenem Melanom, die bis zu 4 Dosen Ipilimumab erhalten hatten, zeigte nach 2 Zyklen (Woche 7) einen signifikanten Unterschied im Gesamtüberleben in Abhängigkeit von der zu diesem Zeitpunkt gemessenen Lymphozytenzahl. Während Patienten mit einer Lymphozytenzahl $\geq 1000 / \mu l$ ein medianes Gesamtüberleben von 11,9 Monaten erreichten, betrug dieses bei niedrigeren Lymphozytenzahlen 1,4 Monate ( $\mathrm{p}<0,0001)$; die entsprechenden 6- und 12-Monats-Überlebensraten betrugen $75 \%$ versus $0 \%$ bzw. $47 \%$ versus $0 \%$ [5].

ICOS und die Lymphozytenzahl geben somit erste Hinweise auf potenziell wichtige Verlaufsparameter, als Entscheidungshilfe für den klinischen Alltag sind sie jedoch nicht geeignet. Hierzu sind weitere Untersuchungen erforderlich.

\section{PD-1/PD-L1-Blockade}

Ein weiteres vielversprechendes Ziel für eine immunologische Tumortherapie ist die Blockade des durch humane Melanomzellen exprimierten Liganden PD-L1 (programmed death li-

\section{KARGER}

Fax +497614520714

Information@Karger.de

www.karger.com
(C) 2012 S. Karger GmbH, Freiburg

0378-584X/12/3514-0002\$38.00/0

Accessible online at:

www.karger.com/onk 
Abb. 1. Phase-IIIStudie MDX010-20: Gesamtüberleben bei Patienten mit vorbehandeltem Melanom im Stadium IIIc/IV (modifiziert nach [2]).

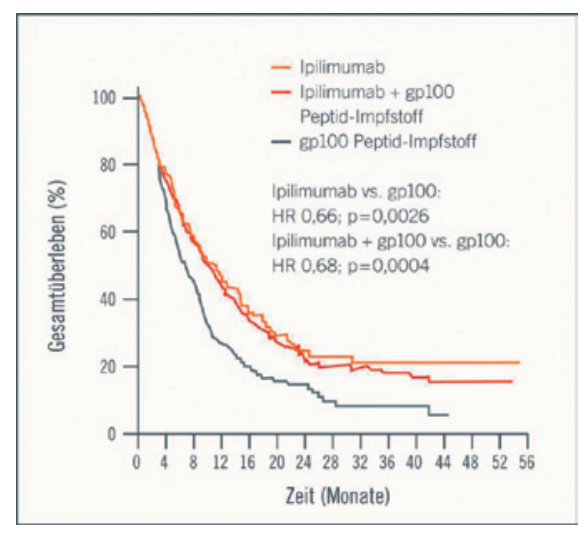

gand 1) bzw. des entsprechenden Rezeptors PD-1, der auf tumorinfiltrierenden Lymphozyten (TIL) stärker exprimiert wird als auf peripheren Blutlymphozyten (PBL) [6]. Da die Interaktion von PD-1 mit seinem Liganden PD-L1 bei einer CTLA-4Blockade zur Einschränkung der T-Zell-Proliferation und Zytokinproduktion führen kann, wurde in tierexperimentellen Studien der Einfluss einer PD-1/PD-L1- und CTLA-4-Blockade auf die Immunantwort untersucht. Im B16-MelanomModell war die Wirksamkeit der Immuntherapie am größten, wenn die Tiere zusätzlich zu einer den Flt3-Liganden exprimierenden Tumorvakzine (FVAX) mit Antikörpern gegen PD-1, PD-L1 und CTLA-4 behandelt wurden (Abb. 2) [7]. Basierend auf diesen Daten wird bei der weiteren klinischen Entwicklung von PD-1- bzw. PD-L1-Antikörpern auch die Kombination mit einem CTLA-4-Antikörper untersucht.

Da in experimentellen Studien auch monotherapeutische Ansätze einer PD-1/PD-L1-Blockade eine vielversprechende Wirksamkeit zeigten, wurde der PD-1-Antikörper BMS936558/ONO-4538 in einer Dosiseskalationsstudie bei Patienten mit verschiedenen fortgeschrittenen soliden Tumoren, darunter auch Patienten mit Melanom, untersucht. Die mit Cut-off vom Dezember 2009 ausgewerteten Daten von 16 Patienten zeigten neben einem günstigen Verträglichkeitsprofil eine hohe Remissionsrate von 37,5\% [8].

\section{Zielgerichtete Therapie}

Der bei Patienten mit nachgewiesener BRAF-V600E-Mutation zum Einsatz kommende BRAF-Inhibitor Vemurafenib reduzierte in einer frühen Interimauswertung der Phase-IIIStudie BRIM3 das Mortalitätsrisiko um 63\% [9, 10]. Da allerdings ein Teil der Melanome relativ schnell gegenüber BRAF-Inhibitoren resistent wird, sind für einen langfristigen Therapieerfolg Strategien zur Überwindung von BRAFResistenzen erforderlich. Einen vielversprechenden Ansatz ermöglichte die Beobachtung, dass bei einer erworbenen BRAF-Resistenz CRAF hochreguliert wird und die Tumorproliferation über CRAF-vermittelte Signalwege angetrieben wird. Tumorzellen, die gegenüber dem selektiven BRAFInhibitor AZ628 resistent waren und erhöhte CRAF-Spiegel
Abb. 2. B16-Melanom-Modell: Einfluss verschiedener Immuntherapien auf das Gesamtüberleben (modifiziert nach [7]).

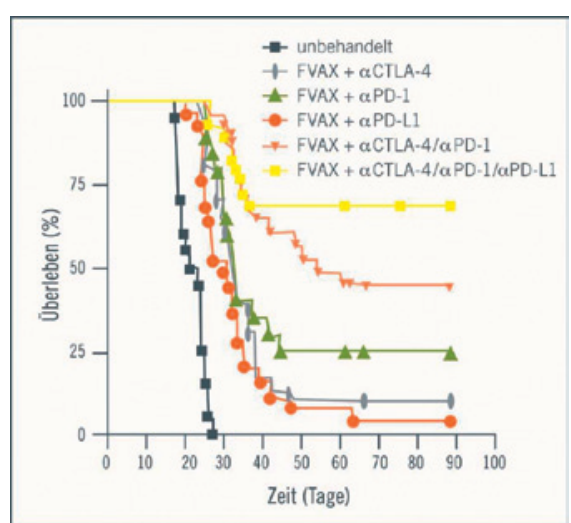

aufwiesen, waren in Zellkultur empfindlich gegenüber dem Heat Shock Protein 90 (HSP90)-Inhibitor Geldanamycin, der damit möglicherweise zur Überwindung einer erworbenen BRAF-Resistenz beitragen kann [11].

\section{Kombinationstherapien}

Ein weiterer Ansatz zur Vermeidung/Überwindung des bei der zielgerichteten Therapie des Melanoms hohen Resistenzrisikos ist die gleichzeitige Gabe eines immunologisch wirksamen Arzneimittels. Als besonders vielversprechend gilt die kombinierte BRAF- und CTLA-4-Blockade, die seit Ende 2011 in einer Phase-I/II-Studie untersucht wird [12].

\section{Sind Rückschlüsse von der metastasierten Situation auf die Adjuvanz möglich?}

Die zuletzt erzielten Fortschritte bei der Behandlung von Patienten mit metastasiertem Melanom legen nahe, die neuen Substanzen auch in der adjuvanten Therapie zu untersuchen. Wie die Erfahrungen bei anderen soliden Tumoren zeigen, lässt sich der Erfolg einer adjuvanten Therapie nur sehr schwierig anhand der Wirksamkeit in der metastasierten Situation vorhersagen. In diesem Zusammenhang wurden exemplarisch verschiedene Systemtherapien bei soliden Tumoren verglichen.

\section{Klassische Chemotherapie}

\section{Nichtkleinzelliges Lungenkarzinom}

Die klassische zytostatische Chemotherapie stellt in der palliativen Therapie von nichtkleinzelligen Lungenkarzinomen (NSCLC) bei den meisten Patienten die Therapie der Wahl dar. In einer großen Metaanalyse, in der die Daten von mehr als 2700 Patienten mit fortgeschrittenem NSCLC ausgewertet wurden, reduzierte die Erstlinien-Therapie mit einer Chemotherapie in Kombination mit einer Supportivtherapie (SC) das Mortalitätsrisiko im Vergleich zur alleinigen SC statistisch signifikant um 23\% (Hazard Ratio (HR) 0,77; p < 0,0001) und verlängerte das mediane Gesamtüberleben von 4,5 auf 6,0 Mo- 
nate bei einer Zunahme des 1-Jahres-Gesamtüberlebens von $20 \%$ auf 29\% [13]. Der Vergleich von 4 platinhaltigen Kombinationstherapien bei chemotherapeutisch nicht vorbehandelten Patienten mit fortgeschrittenem NSCLC zeigte ein medianes Gesamtüberleben von 7,4-8,1 Monaten bei einem 1-Jahres-Gesamtüberleben von 31-36\% [14]. Die adjuvante Therapie mit Vinorelbin/Cisplatin reduzierte in der ANITA-Studie bei Patienten im Stadium IB-IIIA das Mortalitätsrisiko um $20 \%$ (HR 0,80; $\mathrm{p}=0,017$ ), wobei die 5-Jahres-Überlebensrate allerdings nur um $8,6 \%$ verbessert wurde [15].

\section{Kolorektalkarzinom}

Beim metastasierten Kolorektalkarzinom (CRC) lassen sich durch Erstlinien-Schemata unter Einbeziehung von Irinotecan oder Oxaliplatin mediane Überlebenszeiten von 15 Monaten erreichen; im Vergleich zu LV/5-FU (Folinsäure/ Fluorouracil)-Schemata entspricht dies einer Zunahme um etwa 4 Monate. Vergleichbar gute Ergebnisse lassen sich auch in der adjuvanten Situation erzielen, in der FOLFOX (Folinsäure, Fluorouracil, Oxaliplatin) das krankheitsfreie Überleben um etwa $10 \%$ stärker verbessert als LV/5-FU.

\section{Zielgerichtete Therapie mit Angiogenesehemmern}

\section{Kolorektalkarzinom}

Die Zugabe des vaskulären epithelialen Wachstumsfaktor (VEGF)-Antikörpers Bevacizumab zu IFL (Irinotecan, Fluorouracil, Folinsäure) verlängerte beim metastasierten CRC das mediane Gesamtüberleben von 15,6 Monaten (IFL) auf 20,3 Monate [16]. Die adjuvante Therapie mit FOLFOX und Bevacizumab über 12 Monate zeigte dagegen keinen Überlebensvorteil im Vergleich zur alleinigen Chemotherapie [17].

\section{Zielgerichtete Therapie bei selektierten Patienten}

\section{Nichtkleinzelliges Lungenkarzinom}

Asiatische Patienten mit fortgeschrittenem AdenokarzinomNSCLC und nachgewiesener aktivierender Mutation des epidermalen Wachstumsfaktorrezeptor (EGFR)-Gens profitierten in einer Phase-III-Studie signifikant stärker von der Erstlinien-Therapie mit dem EGFR-Tyrosinkinase-Inhibitor (TKI) Gefitinib als von einer Chemotherapie mit Carboplatin/Paclitaxel. Im Vergleich zu Patienten ohne nachgewiesene EGFR-Mutation, die mit dem EGFR-TKI behandelt wurden, war bei den EGFR-Mutation-positiven Patienten unter Gefitinib sowohl die Ansprechrate als auch das progressionsfreie Überleben deutlich verbessert [18].

Vor diesem Hintergrund enttäuschend waren dagegen die Daten zur adjuvanten Therapie mit Erlotinib oder Gefitinib beim EGFR-Mutation-positiven NSCLC. Im Vergleich zu Patienten ohne adjuvante EGFR-TKI-Therapie war der Vorteil im krankheitsfreien und Gesamtüberleben nicht signifikant [19].

\section{Mammakarzinom}

Bei Patientinnen mit HER2 überexprimierendem, metastasiertem Mammakarzinom verbesserte die Zugabe des HER2Antikörpers Trastuzumab zu einem Standard-ErstlinienSchema im Vergleich zur alleinigen Chemotherapie statistisch signifikant das mediane progressionsfreie Überleben (7,4 vs. 4,6 Monate; $\mathrm{p}<0,01$ ), die Remissionsrate ( $50 \%$ vs. $32 \%$; $\mathrm{p}<0,001)$ und das mediane Gesamtüberleben $(25,1$ vs. 20,3 Monate; $\mathrm{p}=0,01$ ) [20].

Ein statistisch signifikanter Vorteil von Trastuzumab zeigte sich auch in der adjuvanten Situation. In der HERAStudie, in der Patientinnen mit HER2-positivem Mammakarzinom nach adjuvanter Chemotherapie 1 oder 2 Jahre mit Trastuzumab behandelt wurden, verbesserte die adjuvante Therapie mit dem HER2-Antikörper (1 Jahr) im Vergleich zur alleinigen Beobachtung das krankheitsfreie 4-JahresÜberleben auf 78,6\%, gegenüber $72,2 \%$ bei Beobachtung (HR 0,76; $\mathrm{p}<0,0001$ ). Bei einer hohen Crossover-Rate von $52 \%$ war das 4-Jahres-Gesamtüberleben nicht signifikant unterschiedlich [21].

\section{Gastrointestinale Stromatumoren}

Patienten mit c-Kit-positivem, metastasiertem gastrointestinalen Stromatumor (GIST) erreichten bei Behandlung mit dem TKI Imatinib, der unter anderem die Tyrosinkinase von c-Kit hemmt, ein medianes progressionsfreies Überleben von 24 Monaten und ein medianes Gesamtüberleben von 55 Monaten [22]. Im Vergleich zu historischen Kontrollen, die chemotherapeutisch behandelt wurden, bedeutet dies eine 4- bis 6-fache Zunahme des medianen Gesamtüberlebens.

Eine signifikante Verbesserung des rezidivfreien Überlebens zeigte Imatinib auch in der ersten randomisierten, placebokontrollierten Studie zur adjuvanten Therapie c-Kit-positiver GIST, in der die 1-jährige Imatinib-Behandlung das Rezidivrisiko signifikant um $65 \%$ reduzierte (HR 0,35; p < 0,0001). Nach Beendigung der Imatinib-Therapie hatten allerdings auch die bisher rezidivfreien Patienten ein hohes Rezidivrisiko (Abb. 3) [23].

In der Phase-III-Studie SSGXVIII/AIO wurde daher bei Patienten mit hohem Rezidivrisiko die Wirksamkeit von Imatinib bei längerer Therapiedauer untersucht. Im Vergleich zur 1-jährigen Behandlung verbesserte die 3-jährige adjuvante Therapie mit Imatinib statistisch signifikant das rezidivfreie Überleben nach 5 Jahren (65,6\% vs. 47,9\%) und das 5-JahresGesamtüberleben (92,0\% vs. $81,7 \%$ ); eine Heilung war aber auch bei der längeren Therapiedauer nicht zu verzeichnen, wie das nach Therapieende ebenfalls erhöhte Rezidivrisiko zeigte [24].

\section{Fazit}

Wirksame Therapien in der metastasierten Situation weisen häufig auch bei adjuvantem Einsatz einen klinischen Vorteil auf. Dabei ist allerdings unklar, welche der bei fortgeschritte- 
Tab. 1. Vergleich Radiosensitizing mit spatialer Kooperation

\begin{tabular}{lll}
\hline & Radiosensitizing & Spatiale Kooperation \\
\hline Prinzip & Verringerung der intrinsischen Radioresistenz des Tumors & Targeting unterschiedlicher Zellpopulationen \\
& Veränderung des Tumor-Mikromilieus & systemische Therapie gegen (Mikro-)Metastasen \\
& Verringerung der Repopulation & Radiotherapie gegen (Primär-)Tumor \\
Vorteile und Nachteile & kurze Behandlungsdauer & längere Behandlungsdauer \\
& Verstärkung radiogener Nebenwirkungen & bessere Verträglichkeit durch sequenzielle Therapie \\
Indikationen (Auswahl) & Kopf-Hals-Tumoren & Leukämien \\
& NSCLC & kindliche Tumoren \\
& Rektumkarzinom & Mammakarzinom \\
\hline
\end{tabular}

Abb. 3. Studie Z9001: Rezidivfreies Überleben (modifiziert nach [23]).

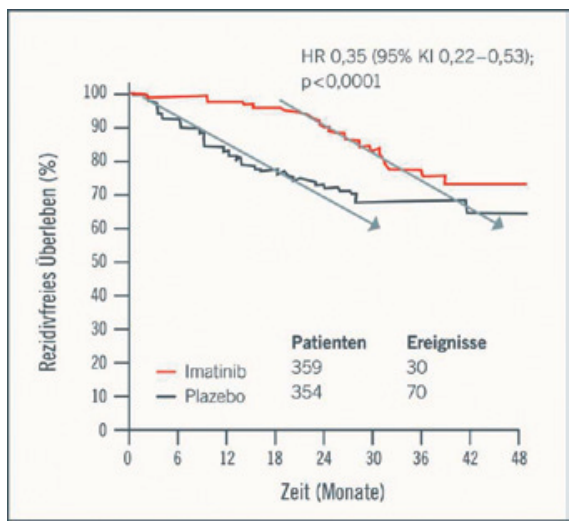

nen Tumoren bekannten arzneimittel- und/oder tumorspezifischen Faktoren für einen Therapieerfolg in frühen Tumorstadien prädiktiv sind. Diskutiert werden unter anderem eine hohe Remissionsrate (vs. niedrige Remissionsrate oder Krankheitsstabilisierungsrate), eine zielgerichtete Therapie mit einem breit wirksamen Angiogenesehemmer (vs. zielgerichtete Therapie mit spezifisch wirksamem Kinase-Inhibitor oder Antikörper) oder ein stabiler Karyotyp des Tumors, wie beispielsweise bei gastrointestinalen Stromatumoren (vs. komplexer Karyotyp wie z.B. beim NSCLC).

\section{Neue Medikamente in Kombination mit Strahlentherapie}

Ansätze zur Verbesserung der Tumorantwort durch Kombination von Medikamenten mit Strahlentherapie betreffen neben dem initialen DNA-Schaden und der DNA-Reparatur vor allem die Tumorhypoxie und die Antigenpräsentation. Bei der Interaktion zwischen Arzneimittel und Radiotherapie wird zwischen Radiosensitizing und spatialer Kooperation unterschieden (Tab. 1).

\section{Radiosensibilisierung durch EGFR-Antikörper}

Der auf verschiedenen soliden Tumoren, darunter Kopf-HalsTumoren, exprimierte EGFR wird durch Bestrahlung aktiviert; über verschiedene Signalkaskaden kann hierdurch nicht nur die Proliferation und das Überleben von Tumorzellen gefördert, sondern auch eine Radioresistenz ausgelöst werden.
Abb. 4. EORTC 26981/NCIC22981: Gesamtüberleben bei adjuvanter Radiochemotherapie oder Radiotherapie (modifiziert nach [26]).

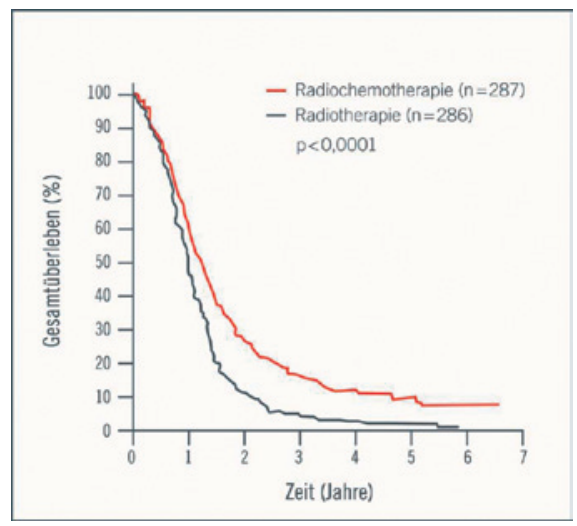

Die Blockade des EGFR mit einem monoklonalen Antikörper verbessert die Radiosensitivität EGFR exprimierender Tumoren und kann das klinische Outcome verbessern. Bei Patienten mit lokal fortgeschrittenen Kopf-Hals-Tumoren führte die Kombination des EGFR-Antikörpers Cetuximab mit einer Strahlentherapie im Vergleich zur alleinigen Bestrahlung zu einer signifikanten Verlängerung der medianen lokoregionalen Kontrolle (24,4 vs. 14,9 Monate; HR 0,68; $\mathrm{p}=0,005)$ und des medianen Gesamtüberlebens (49,0 vs. 29,3 Monate; HR 0,74; $\mathrm{p}=0,03$ ) [25].

\section{Einfluss auf Tumorstammzellen}

Beim Glioblastoma multiforme entwickelt sich nach Abschluss einer Chemotherapie oder Strahlentherapie üblicherweise schnell ein Rezidiv. Als Grund wird das Vorliegen von Tumorstammzellen diskutiert, die durch eine Monotherapie nicht erfasst werden, möglicherweise aber auf eine multimodale Therapie unter Einschluss eines Radiosensitizers ansprechen. In einer Phase-III-Studie mit Patienten, die nach Resektion eines Glioblastoms eine adjuvante Therapie erhielten, konnte durch eine simultane Radiochemotherapie mit Temozolomid im Vergleich zur alleinigen Strahlentherapie das mediane Gesamtüberleben von 12,1 Monaten (alleinige Strahlentherapie) auf 14,6 Monate verlängert werden, wobei das Mortalitätsrisiko signifikant um $37 \%$ abnahm (HR 0,63; p < 0,001). Bemerkenswert war in dieser Studie zudem die bei simultaner Radiochemotherapie relativ hohe Rate der mindestens 2 Jahre überlebenden Patienten (27,2\% vs. 10,9\%) [26] (Abb. 4). 


\section{Beeinflussung der Tumorhypoxie mit dem VEGF- Antikörper}

Ursachen der unter anderem auch beim malignen Melanom $\mathrm{zu}$ beobachtenden Hypoxie sind vor allem arteriovenöse Shunts (AV-Shunts) und temporäre Gefäßverschlüsse, beispielsweise durch Mikroembolien. Da die in hypoxischen Tumorarealen gestörte Vaskularisierung durch Blockierung des VEGF normalisiert werden kann, lässt die Gabe des VEGF-Antikörpers Bevacizumab eine verbesserte Wirksamkeit der Strahlentherapie erwarten. Klinisch untersucht wurde dieser Therapieansatz in einer Phase-II-Studie bei Patienten mit Weichteilsarkom, die präoperativ eine BevacizumabMonotherapie gefolgt von einer simultanen Strahlen-Bevacizumab-Therapie erhielten. Die Integration des VEGF-Antikörpers in das Behandlungsschema ermöglichte eine im Vergleich zu historischen Kontrollen deutliche Verbesserung der Tumorkontrolle, wobei sich auch Blutfluss und Blutvolumen normalisierten [27].

\section{Immunologische Interaktionen}

Experimentellen Daten zufolge kann eine Strahlentherapie die Expression von Major Histocompatibility Complex (MHC)-Klasse-I-Antigenen erhöhen, wobei für diesen Effekt wahrscheinlich hohe Einzeldosen erforderlich sind. Für zukünftige Therapieansätze relevant könnte dabei die Beobachtung sein, dass mit steigender Strahlendosis der relative Anteil radioresistenter regulatorischer T-Zellen $\left(T_{\text {Reg }}\right)$ im Tumor zunimmt. Während eine alleinige Strahlentherapie im Mausmodell das Tumorwachstum nicht beeinflusste, führte die Strahlentherapie in Kombination mit einem gegen $\mathrm{T}_{\mathrm{Reg}}$ gerichteten Antikörper zu einer deutlichen Verzögerung des Tumorwachstums [28].

\section{Zusammenfassung}

Das Therapieprinzip des Radiosensitizing zielt bisher auf die Erhöhung des strahleninduzierten DNA-Schadens oder auf die DNA-Reparatur ab. Ziele der neueren Entwicklungen sind Veränderungen des «Cell Signaling» nach Radiotherapie, der Hypoxie, der Antigenpräsentation und der Immunabwehr.

\section{Disclosure Statement}

Die Autoren waren Referenten beim «2. Interdisziplinären Expertenforum Hautkrebs», das von Bristol-Myers Squibb veranstaltet und unterstützt wurde.

\section{Literatur}

1 Van Elsas A, Hurwitz AA, Allison JP: Combination immunotherapy of B16 melanoma using anti-cytotoxic $\mathrm{T}$ lymphocyte-associated antigen 4 (CTLA-4) and granulocyte/macrophage colony-stimulating factor (GM-CSF)-producing vaccines induces rejection of subcutaneous and metastatic tumors accompanied by autoimmune depigmentation. J Exp Med 1999;190: $355-366$.

2 Hodi FS, O’Day SJ, McDermott DF, et al.: Improved survival with ipilimumab in patients with metastatic melanoma. N Engl J Med 2010;363:711-723.

-3 Schwartzentruber DJ, Lawson DH, Richards JM, et al.: gp100 peptide vaccine and interleukin-2 in patients with advanced melanoma. N Engl J Med 2011; 64:2119-2127.

$4 \mathrm{Fu} \mathrm{T}, \mathrm{He}$ Q, Sharma P, et al.: The ICOS/ICOSL pathway is required for optimal antitumor responses mediated by anti-CTLA-4 therapy. Cancer Res 2011; 71:5445-5454

$5 \mathrm{Ku} \mathrm{GY}$, Yuan J, Page DB, et al.: Single-institution experience with ipilimumab in advanced melanoma patients in the compassionate use setting: lymphocyte count after 2 doses correlates with survival. Cancer 2010;116:1767-1775

6 Blank C, Kuball J, Voelk1 S, et al.: Blockade of PD-L1 (B7-H1) augments human tumor-specific T cell responses in vitro. Int J Cancer 2006;119:317327.

7 Curran MA, Montalvo W, Yagita H, et al.: PD-1 and CTLA-4 combination blockade expands infiltrating $\mathrm{T}$ cells and reduces regulatory $\mathrm{T}$ and myeloid cells within B16 melanoma tumors. Proc Natl Acad Sci USA 2010;107:4275-4280.

8 Sznol M, Powderly JD, Smith DC, et al.: Safety and antitumor activity of biweekly MDX-1106 (antiPD-1, BMS-936558/ONO-4538) in patients with advanced refractory malignancies. J Clin Oncol 2010; 28(suppl 15):abstr 2506.

$\checkmark$ Chapman PB, Hauschild A, Robert C, et al.: Improved survival with vemurafenib in melanoma with BRAF V600E mutation. N Engl J Med 2011:364: 2507-2516.
10 Chapman PB, Hauschild A, Robert C, et al.: Phase III randomized, open-label, multicenter trial (BRIM3) comparing BRAF inhibitor vemurafenib with dacarbazine (DTIC) in patients with ${ }^{\mathrm{V} 600 \mathrm{E}} \mathrm{BRAF}$ melanom J Clin Oncol 2011;29(suppl):abstr LBA4.

11 Montagut C, Sharma SV, Shioda T, et al.: Elevated $\mathrm{CRAF}$ as a potential mechanism of acquired resistance to BRAF inhibition in melanoma. Cancer Res 2008:68:4853-4861.

12 http://clinicaltrials.gov/ct2/show/NCT01400451? term $=n C T 01400451$ and rank $=1$.

13 NSCLC Meta-Analyses Collaborative Group: Chemotherapy in addition to supportive care improves survival in advanced non-small-cell lung cancer: a systematic review and meta-analysis of individual patient data from 16 randomized controlled trials. J Clin Oncol 2008;26:4617-4625.

14 Schiller JH, Harrington D, Belani CP, et al.: Comparison of four chemotherapy regimens for advanced non-small-cell lung cancer. N Engl J Med 2002;346:92-98

15 Douillard JY, Rosell R, De Lena M, et al.: Adjuvant vinorelbine plus cisplatin versus observation in patients with completely resected stage IB-IIIA nonsmall-cell lung cancer (Adjuvant Navelbine International Trialist Association [ANITA]): a randomised controlled trial. Lancet Oncol 2006;7:719-727.

16 Hurwitz H, Fehrenbacher L, Novotny W, et al.: Bevacizumab plus irinotecan, fluorouracil, and leucovorin for metastatic colorectal cancer. N Engl J Med 2004:350:2335-2342.

17 Allegra CJ, Yothers G, O'Connell MJ, et al.: Phase III trial assessing bevacizumab in stages II and III carcinoma of the colon: results of NSABP protocol C-08. J Clin Oncol 2011:29:11-16.

18 Mok TS, Wu YL, Thongprasert S, et al.: Gefitinib or carboplatin-paclitaxel in pulmonary adenocarcinoma. N Engl J Med 2009;361:947-957.

19 Janjigian YY, Park BJ, Zakowski MF, et al.: Impact on disease-free survival of adjuvant erlotinib or gefitinib in patients with resected lung adenocarcinomas that harbor EGFR mutations. J Thorac Oncol 2011;6:569-575.
20 Slamon DJ, Leyland-Jones B, Shak S, et al.: Use of chemotherapy plus a monoclonal antibody against HER2 for metastatic breast cancer that overexpresses HER2. N Engl J Med 2001;344:783-792.

21 Gianni L, Dafni U, Gelber RD, et al.: Treatment with trastuzumab for 1 year after adjuvant chemotherapy in patients with HER2-positive early breast cancer: a 4-year follow-up of a randomised controlled trial. Lancet Oncol 2011;12:236-244.

22 Verweij J, Casali PG, Zalcberg J, et al.: Progressionfree survival in gastrointestinal stromal tumours with high-dose imatinib: randomised trial. Lancet 2004; 364:1127-1134.

23 Dematteo RP, Ballman KV, Antonescu CR, et al. Adjuvant imatinib mesylate after resection of localised, primary gastrointestinal stromal tumour: a randomised, double-blind, placebo-controlled trial. Lancet 2009;373:1097-1104.

24 Joensuu H, Eriksson M, Hartmann J, et al.: Twelve versus 36 months of adjuvant imatinib (IM) as treatment of operable GIST with a high risk of recurrence: Final results of a randomized trial (SSGXVIII/ AIO). J Clin Oncol 2011;29(suppl):abstr LBA1.

25 Bonner JA, Harari PM, Giralt J, et al.: Radiotherapy plus cetuximab for squamous-cell carcinoma of the head and neck. N Engl J Med 2006;354:567-578.

26 Stupp R, Hegi ME, Mason WP, et al.: Effects of radiotherapy with concomitant and adjuvant temozolomide versus radiotherapy alone on survival in glioblastoma in a randomised phase III study: 5-year analysis of the EORTC-NCIC trial. Lancet Oncol 2009:10:459-466.

27 Yoon SS, Duda DG, Karl DL, et al.: Phase II study of neoadjuvant bevacizumab and radiotherapy for resectable soft tissue sarcomas. Int J Radiat Oncol Biol Phys 2011:81:1081-1090.

28 Kachikwu EL, Iwamoto KS, Liao YP, et al.: Radiation enhances regulatory $\mathrm{T}$ cell representation. Int $\mathrm{J}$ Radiat Oncol Biol Phys 2011;81:1128-1135. 